\title{
Launchable rotary wing UAV designs and launch mechanism designs for rotary wing UAV
}

\author{
Etka Gokbel ${ }^{1}$, Sezgin Ersoy ${ }^{2}$ \\ Department of Mechatronics, Marmara University, İstanbul, Turkey \\ ${ }^{1}$ Corresponding author \\ E-mail: ${ }^{1}$ etkagokbel@gmail.com, ${ }^{2}$ sersoy@marmara.edu.tr
}

Received 19 November 2021; received in revised form 6 December 2021; accepted 14 December 2021 DOI https://doi.org/10.21595/jmai.2021.22339

Check for updates

Copyright $(2021$ Etka Gokbel, et al. This is an open access article distributed under the Creative Commons Attribution License, which permits unrestricted use, distribution, and reproduction in any medium, provided the original work is properly cited.

\begin{abstract}
In recent years, rotary-wing unmanned aerial vehicles have been used in many areas. Rotary wing unmanned aerial vehicle (UAV) can carry different payloads according to their duties. For example; if they carry cameras, they are used for reconnaissance / surveillance, cargo if they carry cargo, agriculture if they carry pesticides, mapping if they carry an advanced camera and mapping system, and communication if they carry a base station or relay. Rotary-wing unmanned aerial vehicles are usually commanded to take off manually by a trained UAV operator. Before takeoff, the rotary-wing unmanned aerial vehicle is prepared for take-off by the UAV operator and this preparation takes approximately five minutes. It takes time for rotary-wing unmanned aerial vehicles to take off from the runway and reach their cruising speed, causing time loss in critical areas. A rotary-wing unmanned aerial vehicle launch assembly and a rotary-wing unmanned aerial vehicle with an opening mechanism that can open the thrust arms after launch and continue to fly can be the solution to this time loss. Rotary-wing drones capable of launching and without the intervention of the UAV operator will play an important role in emergency response and defense, where situational awareness is often required. For example; firefighters responding to fires can take advantage of the ability to quickly launch rotary-wing unmanned aerial vehicles from a stationary or moving fire truck. Thanks to the day / thermal camera on the launched rotary wing unmanned aerial vehicles, valuable information can be obtained about the progress of the fire and the damage caused by the fire. Thanks to rapid awareness, the fire can be intervened and fought faster. Similarly, military personnel can quickly deploy launchable rotary-wing drones for reconnaissance and surveillance and perform their duties. In order to be applicable to various types of missions, it is important that the rotary wing unmanned aerial vehicle be portable and low in volume. Since the launchable rotary-wing unmanned aerial vehicle proposed in the thesis has an arm release mechanism after launch, it can automatically open and generate thrust after launching its arms. In this way, it helps lower volume coverage before being launched. This also reduces air friction during launch. It can be deployed to autonomous systems effortlessly as it has closed package, mobile and self-arm management. Different mechanisms will be studied to create an efficient design. A mechanism that allows it to open its arms in a short time will be used in the self-arm-opening management design. Throwable rotary wing unmanned aerial vehicle and launch mechanism will be designed and 3D printers will be used for prototype.
\end{abstract}

Keywords: quadcopter, drone, unmanned aerial vehicle (UAV), vertical take-off and landing (VTOL), bullet-drone, launched UAV, launch mechanism.

\section{Introduction}

Rotary-wing unmanned aerial vehicles play an important role in emergency response and defense, where situational awareness is often required [1]. Because it takes a long time for rotary wing unmanned aerial vehicles to take off, which will provide information for firefighters responding to fires, valuable information about the progress and destruction of the fire may be reached after it is too late. It takes time for rotary wing unmanned aerial vehicles to take off from the ground and reach cruising speed and that cause loss of time in critical areas. If the rotary wing 
unmanned aerial vehicle can be launched rapidly and the mission altitude is reached quickly, the fire can be intervened more quickly.

Some consumer rotary-wing UAV can be folded to take up a small volume of arms, but opening the arms is manual and not designed to be compatible with launch mechanisms. e.g. DJI's Mavic series [2], Parrot's Anafi series [3] and Autel's Evo series [4] are among the most popular UAV companies. The products of all three companies can be folded horizontally by hand to fit in a small volume. GDU's $\mathrm{O} 2$ Plus product is also foldable by hand, but it can be folded by sliding the arms [5]. Another example is the UAV in the quadcopter configuration with an egg-shaped Power Egg from Power Vision company shown in Fig. 1. Its arms can be folded by hand [6].

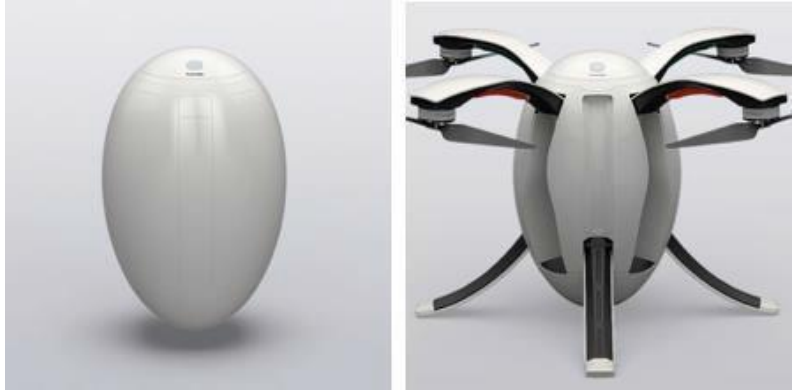

Fig. 1. The product named power egg of power vision company [6]

LeveTop's rotary-wing UAV looks like a small cylinder when folded, and its arms are foldable by hand. The rotary-wing UAV in Fig. 2 is in quadcopter configuration with four-motor [7].

The rotary-wing UAV in Fig. 3, named Sprite, developed by Ascent Aerosystems, has two coaxial motors and resembles a cylinder [8].

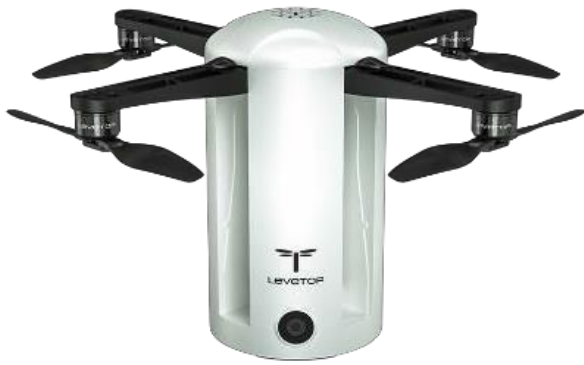

Fig. 2. Product of LeveTop company [7]

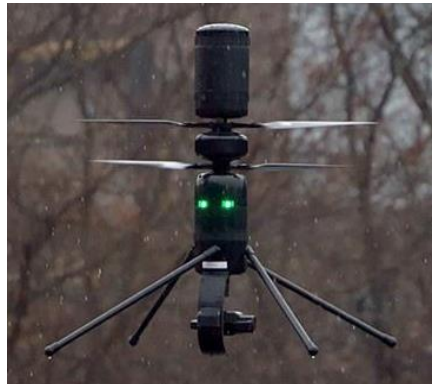

Fig. 3. Ascent Aerosystems product called Sprite [8]

It is mentioned that traditional quadcopter designs do not have the ability to change their configuration to meet the need to adapt to the requirements of the mission. In order to be fully applicable to various types of missions; it is emphasized that it is vital that the unmanned aerial vehicle be portable and have a low volume. It is mentioned that morphological quadcopters can fold and unfold their arms autonomously, as they have self-arm management. Tuna et al. [9] presented a self-folding and self-deployable autonomous quadcopter called Folly. In its self-arm management mechanism design, it features a crank connecting rod mechanism driven by a single servo motor, allowing the Folly to automatically fold and unfold its arms within a short period of time. It takes $600 \mathrm{~ms}$ for the UAV shown in Fig. 4 to go from folded to open.

The quadcopter mentioned by Bucki and Mueller [10] has spring hinges that allow the arms to fold down. A reduction of approximately $50 \%$ was achieved in the folded volume of the UAV in Fig. 5. 


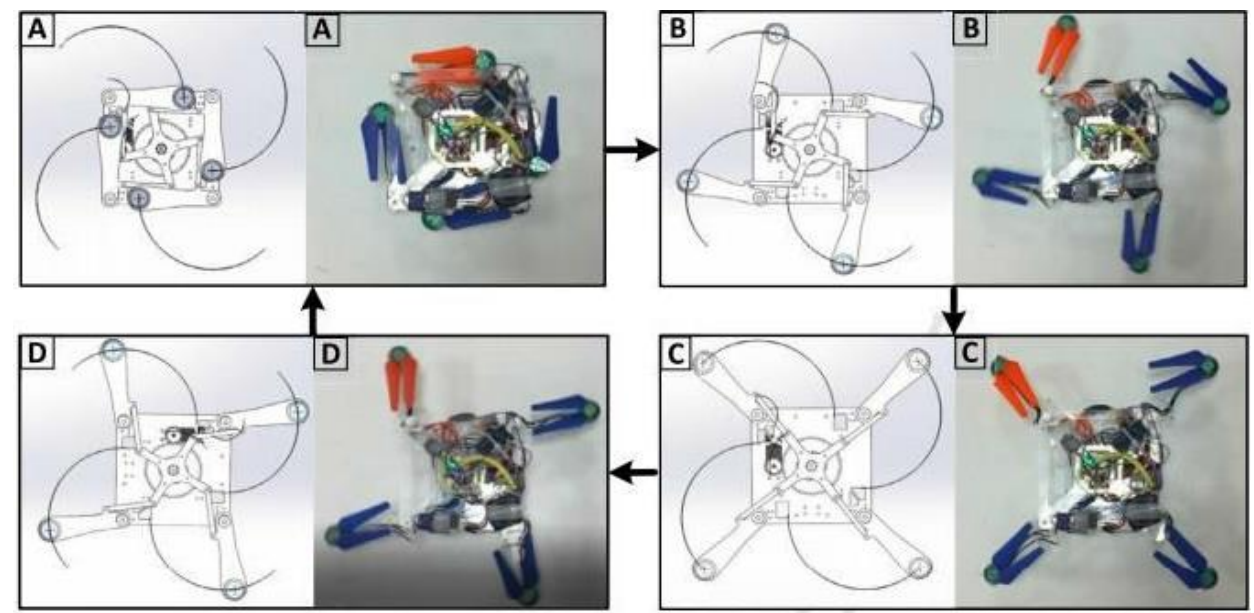

Fig. 4. Foldable drone named Folly [9]

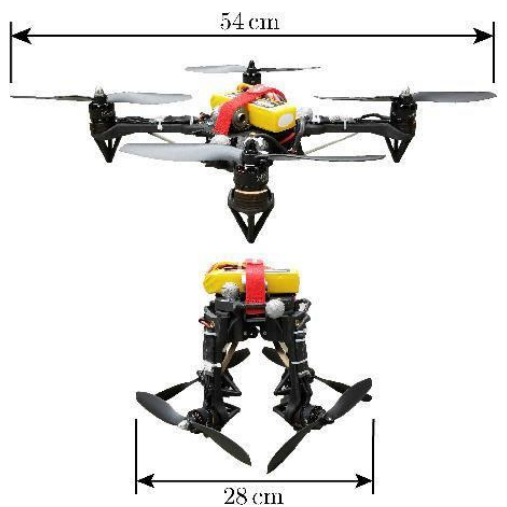

Fig. 5. Shape-shifting quadcopter [10]

Falanga et al. [11] mentioned a rotary wing unmanned aerial vehicle that changes its shape to adapt to the environment. Changing the morphology of the UAV during flight has increased the complexity of the platform and reduced its controllability.
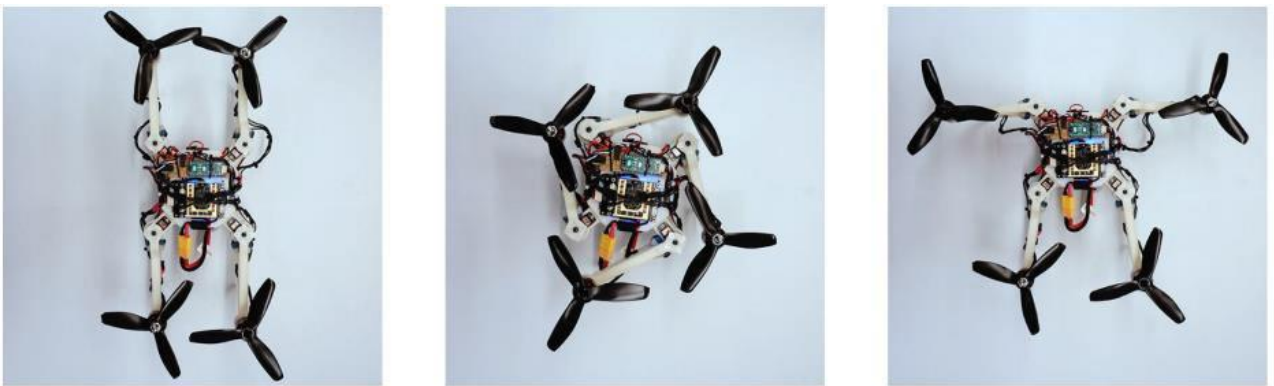

Fig. 6. Morphology change of UAV [11]

Gao et al. [12] mentioned a projectile-shaped vehicle that can both fly in the air and move underwater, with no launch mechanism.

Devlin et al. [13] mentioned a rotary wing unmanned aerial vehicle with a servo motor in each arm. Thanks to this servo motor, each BLDC motor can rotate in an axis perpendicular to the arm. 


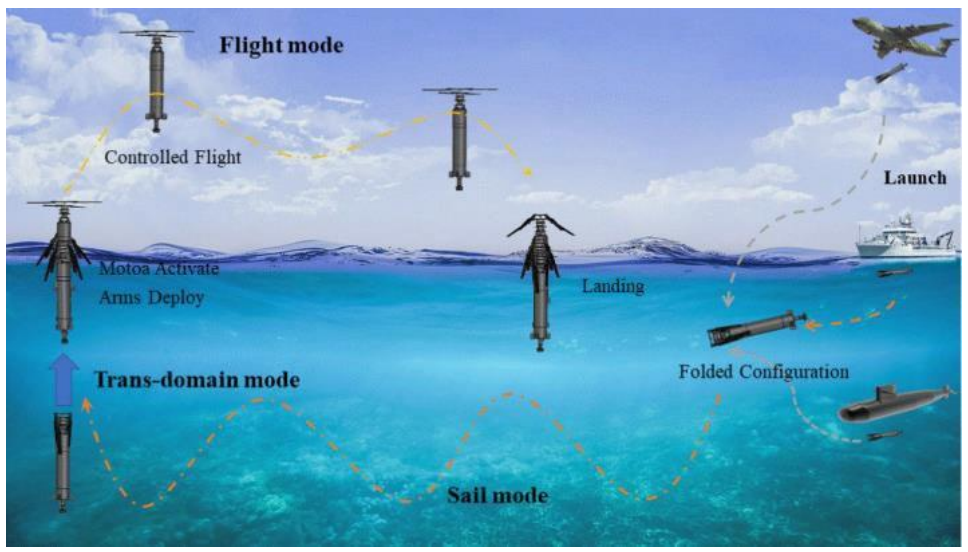

Fig. 7. UAV with amphibious features [12]

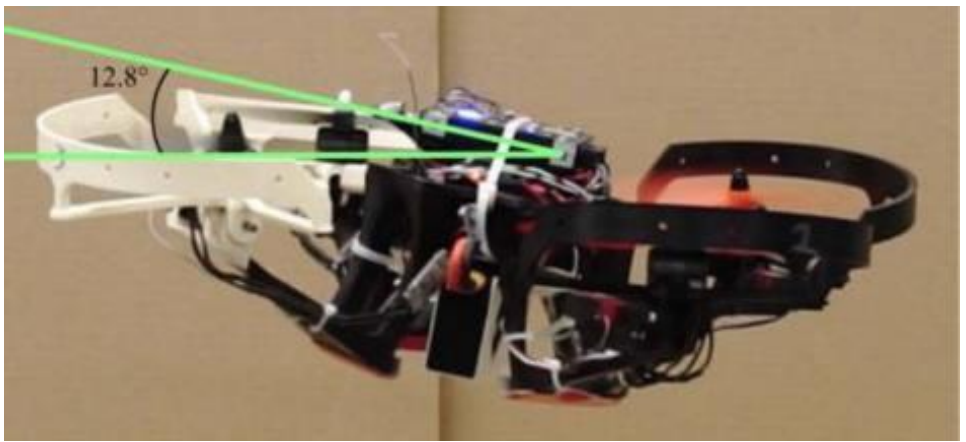

Fig. 8. UAV with an elbow-like rotating motor [13]

These rotary-wing UAVs are not designed for a launch system and therefore cannot be launched.

For example, Spear UAV's solutions are based on encapsulated rotary-wing unmanned aerial vehicles. This company works to manage the chaotic battlefield by producing UAVs that are instantly available and accessible for the end user. Ninox 40 and Ninox 66 products are shown in Fig. 9 [14]. There is no information about the arm opening mechanisms of the products.
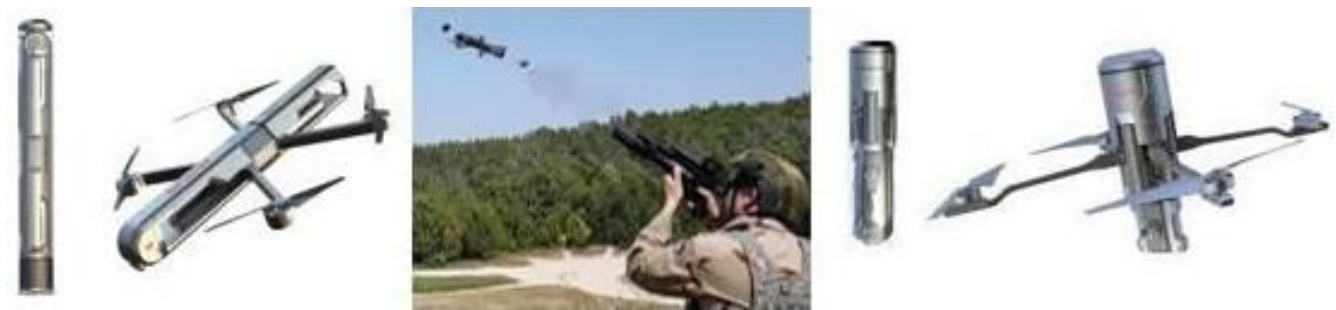

Fig. 9. Ninox 40 and Ninox 66, products of Spear UAV company [14]

Defendtex Company produces unmanned aerial vehicle that can be launched from a $40 \mathrm{~mm}$ grenade launcher. Drone40 has a weight of 190 grams and a range of $20 \mathrm{~km}$ [15].

Gnemmi and Changey [16] introduced a hybrid system with a weight of approximately one $\mathrm{kg}$ for a very fast observation mission with the GLMAV unmanned aerial vehicle. The GLMAV in a projectile configuration, weighing less than $10 \mathrm{~kg}$, is launched through a portable special tube in Figure Fig. 11, and when it reaches the targeted area (at $100 \mathrm{~m}$ altitude and over $500 \mathrm{~m}$ range), it turns on the engine system and propellers. 


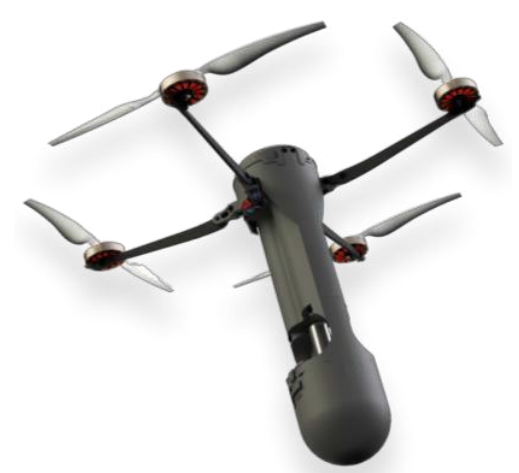

Fig. 10. Defendtex company's DRONE40 product [15]
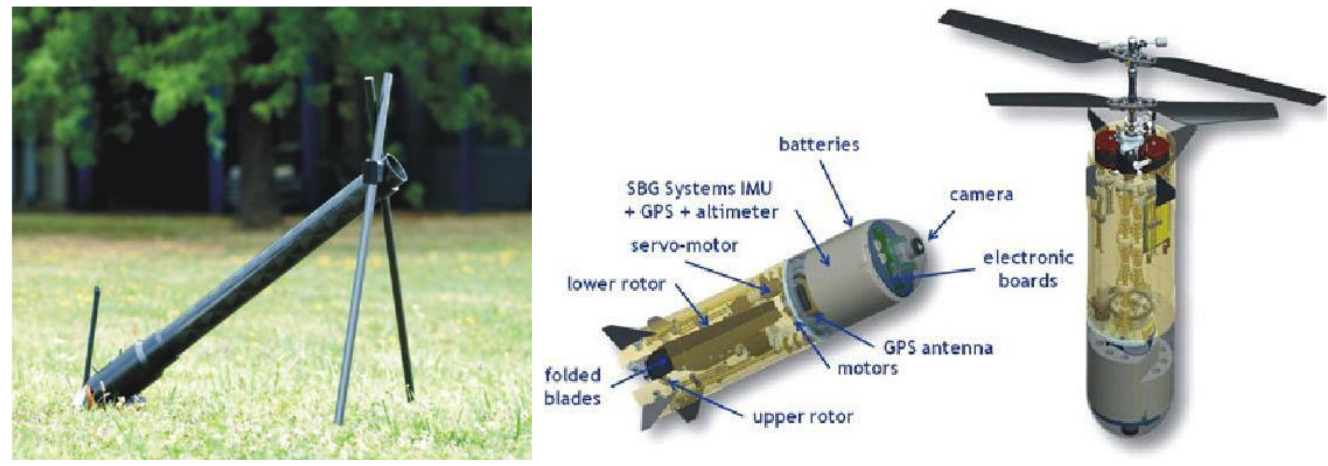

Fig. 11. Bullet drone [16]

Gnemmi et al. [17] described how a packaged micro-UAV (MAV) like a projectile in Fig. 12 is launched using the energy provided by a portable launch weapon.

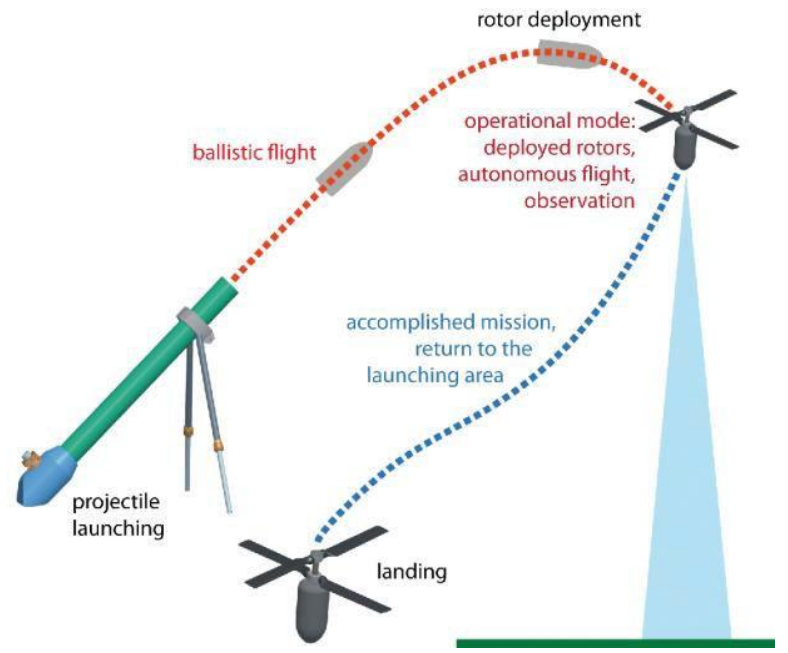

Fig. 12. Unmanned aerial vehicle that can be launched from a gun [17]

There are several products for military applications for fixed-wing UAV. For example, Coyote in Fig. 13 developed by Raytheon is a fixed-wing UAV [18]. 


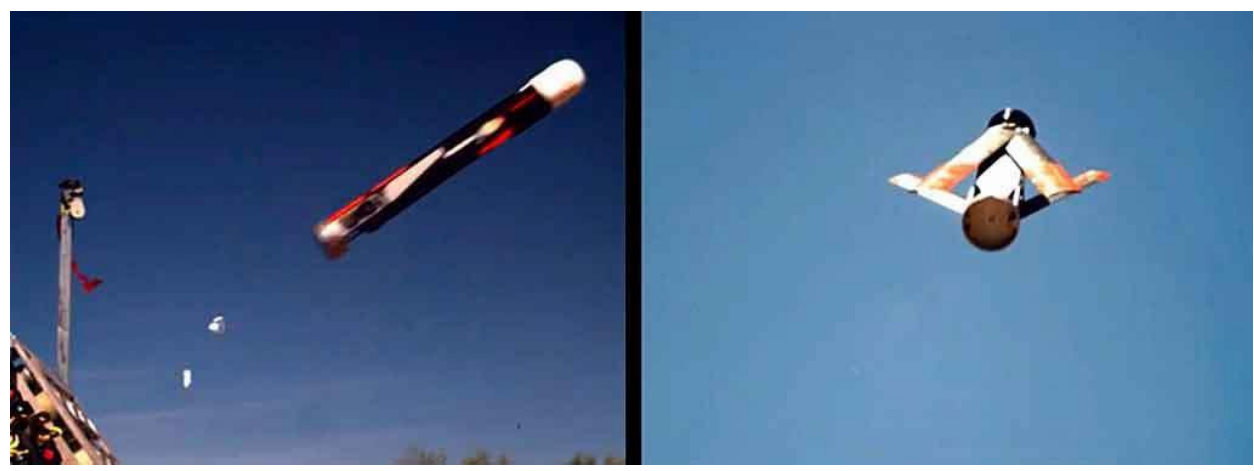

Fig. 13. Raytheon's Coyote product [18]

Developed by the UVision Company, the Hero 30 ISR has a weight of three $\mathrm{kg}$. The system is launched by placing it in a tin can in Fig. 14 [19].

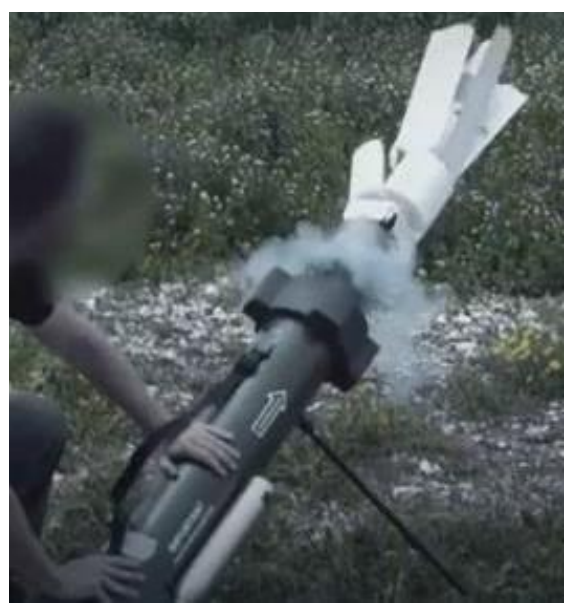

Fig. 14. UVision Company's Hero-30 product [19]

The "Switchblade" developed by AeroVironment is the fixed-wing UAV in Fig. 15 [20].

Developed by OTO Melara, the Horus can be launched from the $120 \mathrm{~mm}$ tank barrel in Fig. 16 or from a customized launch system [21].

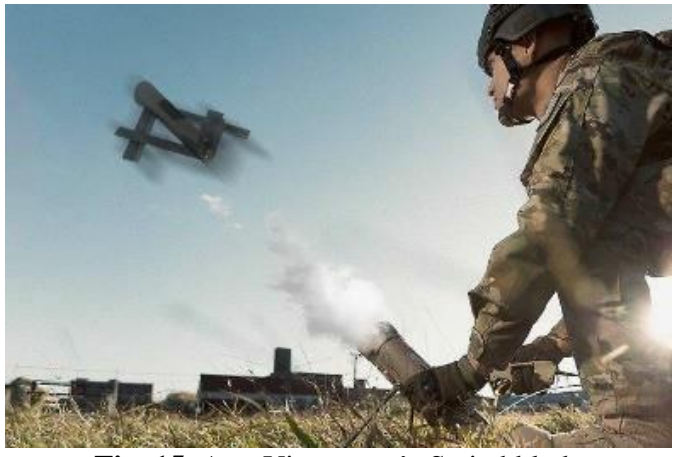

Fig. 15. AeroVironment's Switchblade product [20]

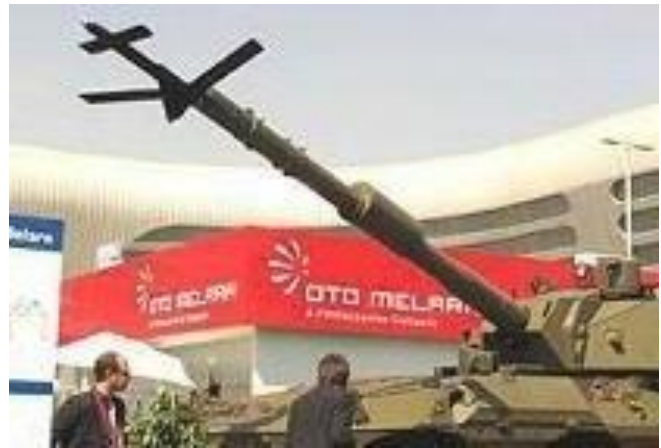

Fig. 16. Horus product of OTO Melara company [21] 


\section{Material and methods}

Since the popular products sold in the market do not have a self-folding / unfolding feature and there is no launch mechanism, it was necessary to design and prototype a new unmanned aerial vehicle. It was necessary to design a rotary-wing unmanned aerial vehicle launch mechanism and a rotary-wing unmanned aerial vehicle with an opening mechanism that could open the arms and produce thrust to continue flying after launch. During launch, the rotary-wing unmanned aerial vehicle changes its shape from an aerodynamic projectile to a multi-rotor. The proposed rotary-wing UAV design is lightweight, aerodynamic, and has multiple moving parts that must fit into a special small-diameter launch barrel.

First, the mechanisms were investigated and shaped. Production with FDM type 3D printing technology was preferred to create a compact, robust and independent platform by taking advantage of the ability to design and produce complex geometries with a short time and low cost budget. The structural design of the prototype was made in a printable way on a 3D printer. The launchable rotary wing unmanned aerial vehicle designed for this study and its launch mechanism are given in Fig. 17.

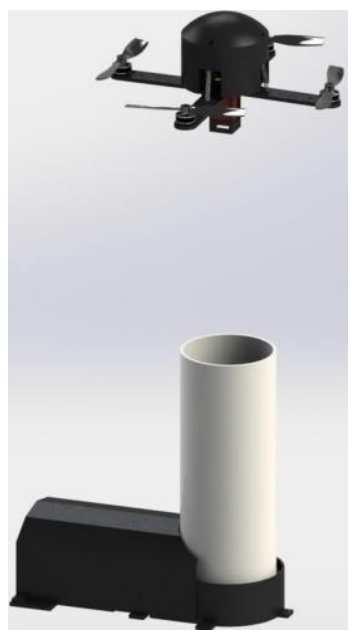

Fig. 17. Proposed launchable rotary wing unmanned aerial vehicle and launch mechanism

\section{Results}

In this section, information is given about the results obtained, the problems encountered and the improvements that can be made. In addition, it has been compared with similar researches in the country and abroad mentioned in the literature review.

Kuperman [22], the inventor of the patent number WO2020144690A1, mentioned the capsule that enables encapsulation of the rotary wing unmanned aerial vehicle. It is mentioned that there is a controller and an actuator to release the capsule. When the conditions are suitable for the UAV to exit the capsule, the UAV is released by the actuator. Since the weight of the capsule and the actuators on it will be added for the launch, the amount of energy required for the launch and also the cost will increase. However, by adjusting the opening of the capsule at the right time, the wind resistance acting by the capsule will be minimal until the UAV reaches the desired altitude.

Guruge et al. [23] presented a Bluetooth integrated catapult launcher system for fixed-wing UAVs in Fig. 18. In the launcher, they used the spring as the energy source and the electromagnetic release system to release the spring. It is a system produced entirely for fixed-wing UAVs. It provides the horizontal speed and thus the lifting force that fixed-wing UAVs need to gain altitude. The system is big. Rotary-wing UAVs need to gain altitude by launching vertically, not horizontally. 


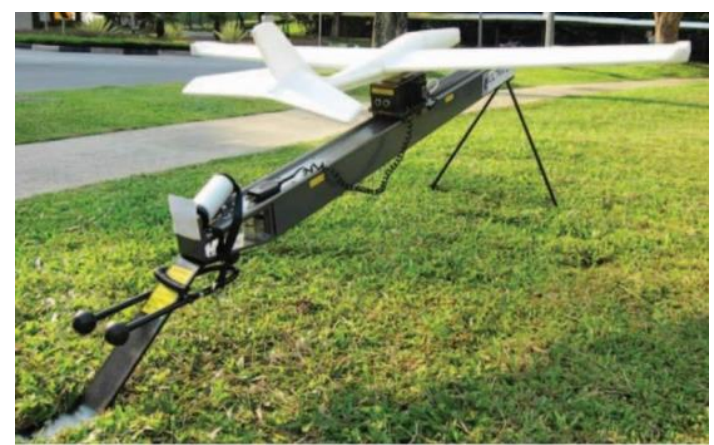

Fig. 18. Spring catapult launcher system (for fixed wing UAVs) [23]

Patterson and Arena [24] stated that launching catapults for fixed-wing UAVs are commercially available, but they can be very expensive. They also mention that some special UAVs need special launch catapults and different launch designs. These are spring systems, pneumatic systems as in Fig. 19, or belt-pulley systems.

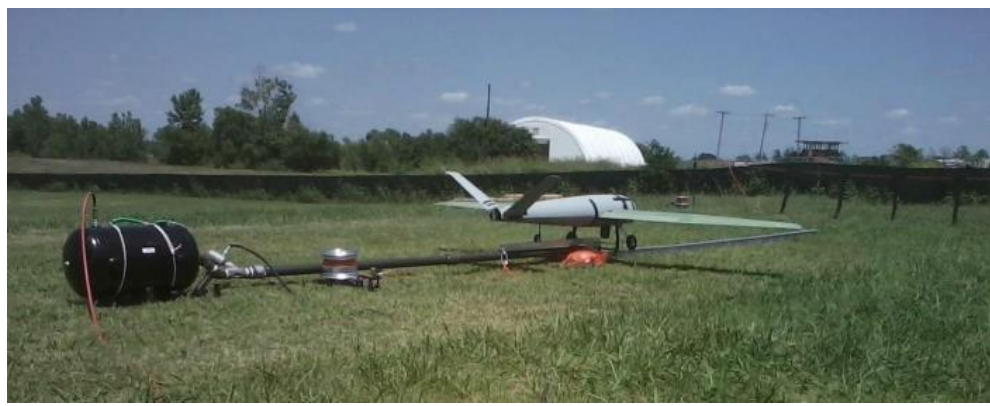

Fig. 19. Pneumatic catapult launcher system (For Fixed Wing UAVs) [24]

Foster et al. [25] introduced a detachable quadcopter from the launched rocket. After the rocket reaches its peak, it is aimed that the quadcopter will leave and return to the take-off point. They mentioned that torsion springs are used as in Fig. 20 to open the arms of the quadcopter.

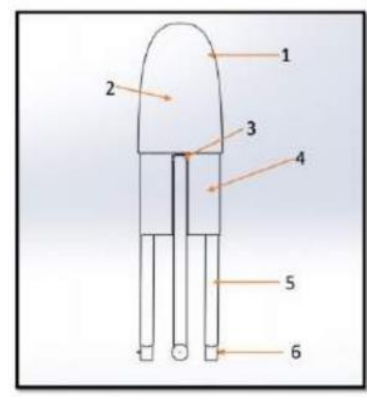

Side View: Arms Retracted

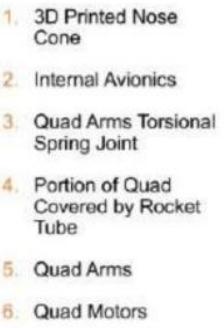

Fig. 20. Torsion spring arm opening mechanism [25]

Down et al. [26] introduced the systems they developed to capture and immobilize a second object in their patent. It is the enemy UAVs that are mentioned as the second object to be captured. The launcher works with high pressure of the gun. In order to catch the target, the net and parachute are thrown from the gun in the capsule. When the capsule arrives at the destination, the capsule leaves the net and parachute and arrives at the destination. Thanks to the capsule, the 
ammunition is provided at the right time, while the weight and cost of the capsule is a disadvantage.

Lee et al. [27] developed a new method to extend the flight time of unmanned aerial vehicles with limited flight time due to the battery. In the proposed method, a carrier foldable rotary-wing UAV is launched with a rocket. When the rocket reaches a certain altitude, the rotary-wing UAV leaves the rocket. In this way, the energy consumed to rise to flight altitude is reduced. The weight of the rocket is $35 \mathrm{~kg}$, the weight of the UAV is $0.61 \mathrm{~kg}$, and the weight of the container that allows to drop the UAV is $0.36 \mathrm{~kg}$. It can be a method for UAVs that will fly at very high altitudes, but rotary wing unmanned aerial vehicles generally do not need to go higher than $1000 \mathrm{~m}$ above ground level. For example, if the rotary-wing UAV carries the camera payload and is above $1000 \mathrm{~m}$ altitude, it needs to carry a very advanced camera with high electro-optical zoom capability.

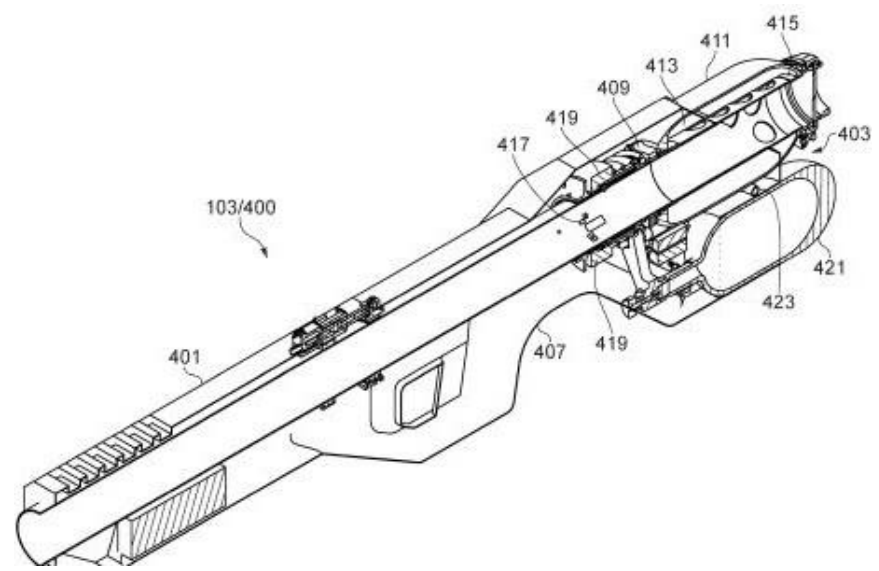

Fig. 21. Pressure operated net gun [26]
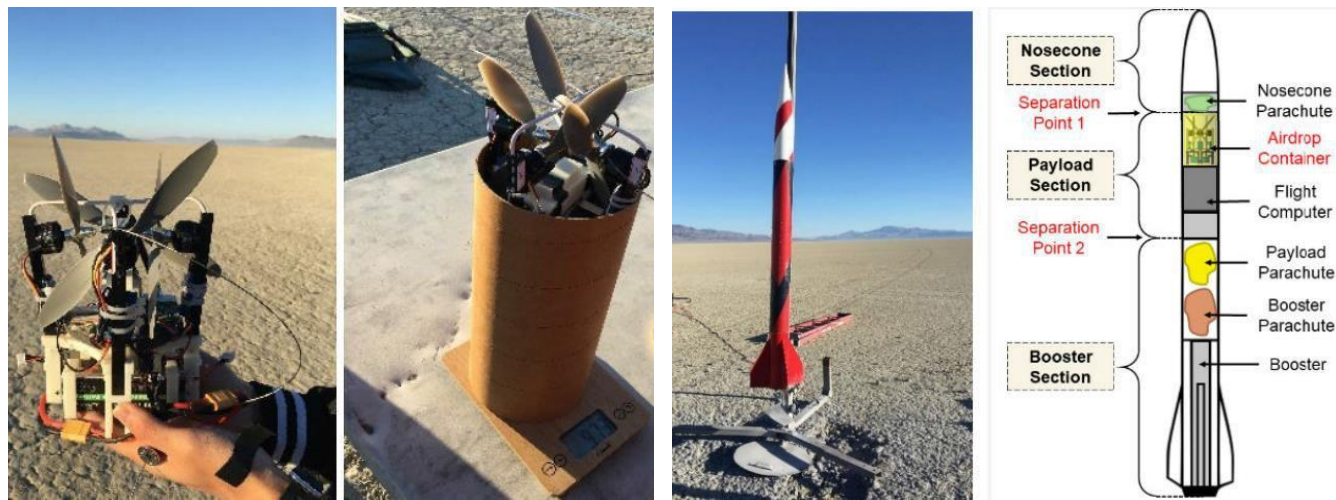

Fig. 22. Rotary wing UAV detachable from rocket [27]

Pastor et al. [28] made a conceptual presentation of the rotary-wing UAV, which can switch from a ballistic launch configuration to a fully controllable flight configuration after launch. A three-inch diameter barrel was used in the study. The launch mechanism is not specific to the system and a pneumatic baseball shooting machine is used. The body, nose and arms of the UAV in Fig. 23 were produced with a 3D printer. 


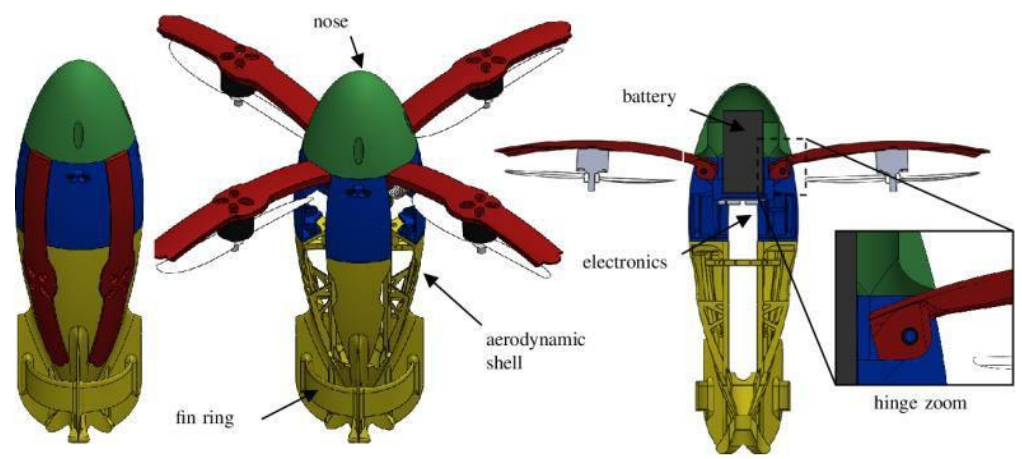

Fig. 23. Ballistic rotary-wing UAV [28]

The weight of the UAV is 530 grams and its launch speed is $15 \mathrm{~m} / \mathrm{s}$. Each arm has a hinge and torsion spring. By using fishing line, it is ensured that the arm remains closed until the desired moment. With the current flowing over the nichrome wire, the release mechanism is triggered and the fishing line melts and opens the propulsion system. The biggest disadvantage of using nichrome wire and line is that the line and nichrome wire need to be replaced after each flight.

Henderson et al. [29] investigated bio-inspired design strategies to guide the structural design of a 3D-printable, foldable and ballistically deployable multi-rotor unmanned aerial vehicle (UAV) platform. UAV analysis, including FEA stress simulations, aerodynamic tests, and mechanical prototypes, to assess feasibility is shown in Fig. 24. Thanks to the solenoid motor in the center of the platform, the bar that keeps the arms closed is released and the arms are opened by springing. Elastic bands provided the opening of the arms. The solenoid motor is responsible for opening the six arms when free fall is detected via the flight software. The presence of the solenoid motor will increase the weight of the UAV, so it has the effect of reducing the flight time of the UAV. It was stated that many methods could be used for launching, from hand throwing to mortar-style barrels, and it was mentioned that the work on the launch mechanism continues and the barrel will be 8 inches.

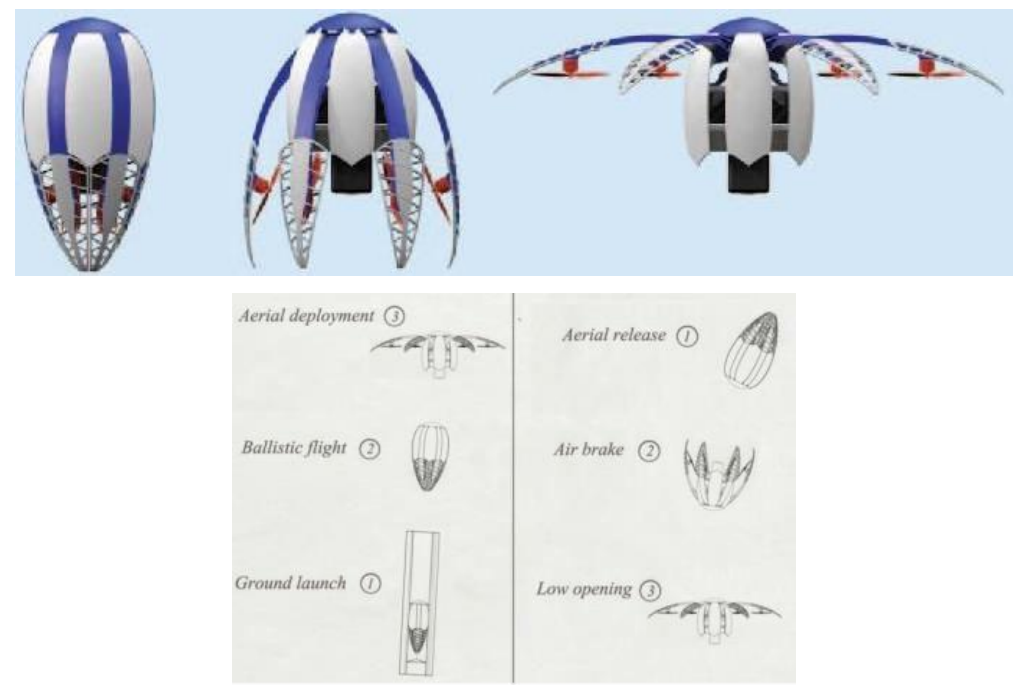

Fig. 24. Bio-inspired Rotary-Wing UAV [29]

Bouman et al. [30] present the SQUID (Streamlined Quick Unfolding Investigation Drone) shown in Fig. 25 in their article where they mention about rotary-wing UAV that can launch ballistically and transform into six rotor rotary wing unmanned aerial vehicle. 


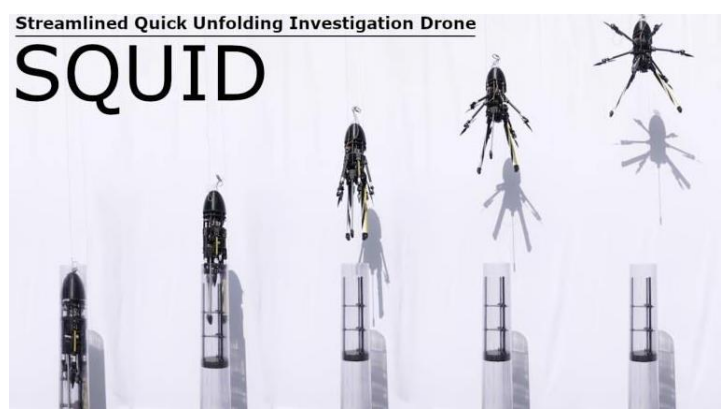

Fig. 25. SQUID Rotary-Wing UAV [30]

The weight of the UAV is $3.3 \mathrm{~kg}$ and its muzzle velocity is $12 \mathrm{~m} / \mathrm{s}$. High pressure carbon dioxide gas, known as T-shirt ball, was used as the launch mechanism. The major disadvantage of pneumatic systems is that gas must be filled before each launch. After the UAV comes out of the launch tube, the thrust arms are opened immediately to the hinges and torsion springs.

\section{Conclusion}

Rotary-wing unmanned aerial vehicles carry different types of payloads according to their mission. E.g. if the advanced imaging unit carries the payload, it performs the reconnaissance surveillance task, if it carries the payload, it performs the cargo transport task, if the repeater relay carries the payload, and it performs the communication task.

In this study, rotary wing unmanned aerial vehicles of the launch mechanism were evaluated.

It is thought that a rotary wing unmanned aerial vehicle launch mechanism and a rotary wing unmanned aerial vehicle with an opening mechanism that can open the thrust arms and continue flying after launch can be a solution to this waste of time. Launchable rotary-wing drones will play an important role in emergency response and defense, where situational awareness is often required.

\section{References}

[1] G. L. Foresti, M. Farinosi, and M. Vernier, "Situational awareness in smart environments: socio-mobile and sensor data fusion for emergency response to disasters," Journal of Ambient Intelligence and Humanized Computing, Vol. 6, No. 2, pp. 239-257, Apr. 2015, https://doi.org/10.1007/s12652-0140227-x

[2] https://store.dji.com/product/dji-mavic-3 (Last accessed: 03.12.2021).

[3] https://www.parrot.com/us/drones/anafi (Last accessed: 03.12.2021).

[4] https://www.autelrobotics.com/productdetail/23.html (Last accessed: 03.12.2021).

[5] http://www.gdu-tech.com/en/o2 (Last accessed: 03.12.2021).

[6] https://www.powervision.me/en/product/poweregg (Last accessed: 03.12.2021).

[7] https://www.levetop.com/ (Last accessed: 03.12.2021).

[8] https://ascentaerosystems.com/defense/(Last accessed: 03.12.2021).

[9] T. Tuna, S. Ertug Ovur, E. Gokbel, and T. Kumbasar, "Design and development of folly: A self-foldable and self-deployable quadcopter," Aerospace Science and Technology, Vol. 100, p. 105807, May 2020, https://doi.org/10.1016/j.ast.2020.105807

[10] N. Bucki and M. W. Mueller, "Design and control of a passively morphing quadcopter," in International Conference on Robotics and Automation (ICRA), 2019.

[11] D. Falanga, K. Kleber, S. Mintchev, D. Floreano, and D. Scaramuzza, "The foldable drone: a morphing quadrotor that can squeeze and fly," IEEE Robotics and Automation Letters, Vol. 4, pp. 209-216, 2019.

[12] Y. Gao, H. Zhang, H. Yang, S. Tan, T. A. Gulliver, and T. Lu, "Trans-Domain Amphibious Unmanned Platform Based on Coaxial Counter-Propellers: Design and Experimental Validation," IEEE Access, Vol. 9, No. 99, pp. 149433-149446, 2021, https://doi.org/10.1109/access.2021.3125138

[13] T. Devlin, R. Dickerhoff, and K. F. A. Durney, "Elbowquad: Thrust vectoring quadcopter," in AIAA Information Systems-AIAA Infotech@Aerospace, 2018. 
[14] https://spearuav.com/solutions/ (Last accessed: 03.12.2021).

[15] https://www.defendtex.com/wp-content/uploads/D40_V10_Product_DataSheet.pdf (Last accessed: 03.12.2021)

[16] P. Gnemmi, S. Changey, K. Meder, E. Roussel, C. Rey, C. Steinbach, and C. D. Berner, "Conception and manufacturing of a projectile-drone hybrid system," IEEE/ASME Transactions on Mechatronics, Vol. 22, No. 2, pp. 940-951, Apr. 2017, https://doi.org/10.1109/tmech.2017.2654018

[17] P. Gnemmi, K. Meder, and C. Rey, "Aerodynamic Performance of a Gun Launched Micro Air Vehicle," International Journal of Micro Air Vehicles, Vol. 4, No. 4, pp. 251-272, Dec. 2012, https://doi.org/10.1260/1756-8293.4.4.251

[18] https://www.raytheonmissilesanddefense.com/capabilities/products/coyote (Last accessed: 03.12.2021)

[19] https://uvisionuav.com/main-products (Last accessed: 03.12.2021).

[20] https://www.avinc.com/tms/switchblade-600 (Last accessed: 03.12.2021).

[21] https://www.leonardocompany.com/en/news-and-stories-detail/-/detail/otomelara-droni-droneseuropean-research-night (Last accessed: 03.12.2021).

[22] G. Kuperman, "Unmanned Aerial Vehicle Launching Capsule," WO2018229747A1, 2020.

[23] P. Guruge, B. B. Kocer, and E. Kayacan, "A novel automatic UAV launcher design by using bluetooth low energy integrated electromagnetic releasing system," in 2015 IEEE Region 10 Humanitarian Technology Conference (R10-HTC), Dec. 2015, https://doi.org/10.1109/r10-htc.2015.7391861

[24] A. J. Patterson and A. S. Arena, "A Launch System Design Methodology for Small Unmanned Aircraft Applications," in AIAA Atmospheric Flight Mechanics Conference, Vol. 2858, Jun. 2015, https://doi.org/10.2514/6.2015-2858

[25] N. Foster, L. Utley, A. Walsh, B. Kadavy, C. Kenkel, G. McCullers, L. Kunka, N. George, C. Ritchie, and J. Rosario, "Rocket launched autonomous quadcopter final report," American Institute of Aeronautics and Astronautics, 2018.

[26] C. D. Down, N. R. Armstrong, J. E. Cross, A. J. Wilkinson, and R. S. Wilkinson, "System for deploying a first object for capturing, immobilising or disabling a second object," GB2538826B, 2016.

[27] C. Lee, S. Lee, and B. Chu, "Extension of quadcopter flight range based on quadcopter transport system and autonomous ramp flight algorithm," IEEE Access, Vol. 8, No. 99, pp. 156422-156432, 2020, https://doi.org/10.1109/access.2020.3019066

[28] D. Pastor, J. Izraelevitz, P. Nadan, A. Bouman, J. Burdick, and B. Kennedy, "Design of a ballisticallylaunched foldable multirotor," in 2019 IEEE/RSJ International Conference on Intelligent Robots and Systems (IROS), Nov. 2019, https://doi.org/10.1109/iros40897.2019.8968549

[29] L. Henderson, T. Glaser, and F. Kuester, "Towards bio-inspired structural design of a 3D printable, ballistically deployable, multi-rotor UAV," in 2017 IEEE Aerospace Conference, pp. 1-7, Mar. 2017, https://doi.org/10.1109/aero.2017.7943970

[30] A. Bouman, P. M. Nadan, M. O. Anderson, D. Pastor, J. S. Izraelevitz, J. W. Burdick, and B. Kennedy, "Design and autonomous stabilization of a ballistically launched multirotor," in IEEE International Conference on Robotics and Automation, May 2020.

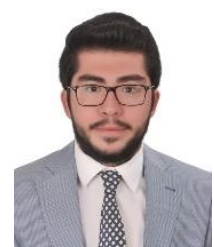

Etka Gokbel received M.Sc. degree in Mechatronics from Marmara University, İstanbul, Turkey, in 2021. He graduated from Istanbul Technical University Mechanical Engineering Department in 2018. His undergraduate project on autonomous foldable quadcopter was supported by TUBITAK. Afterwards, they presented a conference paper on this topic with their friends and was awarded the best paper of the conference. He received a patent on UAV with ITUNOVA. He wrote an article about the foldable quadcopter and was published in the journal Aerospace Science and Technology.

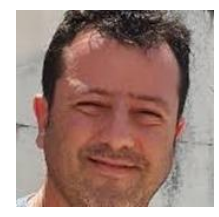

Sezgin Ersoy is an Associate Professor of Mechatronics Engineering and Material Science. After graduating from Marmara University, he became a faculty member at the same university. His publications include a variety of efforts to understand changes in automotive mechatronics, polymer science, and biomedical technologies. He was granted a fellowship at the TUBITAK at Bourgogne University ISAT and spent one year as a visiting fellow there to study several projects between 2014 through 2015 . He is the author of chapter Science Education in a Rapidly Changing World, USA 2011, and the author in Acoustic Properties of Bio-Materials, Stuttgart, 2010. He has two science national awards and is an Editorial Member of several scientific journals. 